\title{
Demographischer Wandel und Gesundheitsausgaben: Theorie, Empirie und Politikimplikationen
}

DOI 10.1515/pwp-2015-0016

Zusammenfassung: In diesem Übersichtsartikel erörtert Friedrich Breyer zunächst theoretisch den Effekt der Alterung und des medizinischen Fortschritts auf die Gesundheits- und Pflegeausgaben und stellt den Stand der empirischen Forschung dar. Dabei hinterfragt er kritisch vor allem die „Red-herring“-Hypothese, nach der die Alterung deshalb kein Ausgabentreiber ist, weil es zu einem großen Teil der Gesundheitsausgaben nur in wenigen Jahren vor dem Tod kommt. Aus den empirischen Ergebnissen lassen sich Prognosen über die Tragfähigkeit der Finanzierung der deutschen Kranken- und Pflegeversicherung ableiten. Breyer erörtert zudem Reformvorschläge für die Krankenund Pflegeversicherung, die helfen könnten, die demographische Herausforderung zu bewältigen, und fragt nach der politischen Durchsetzbarkeit solcher Reformen in einer alternden Gesellschaft. ${ }^{1}$

\section{JEL-Klassifikation: H 51, J 11, I 18}

Schlüsselwörter: Gesundheitsausgaben, Pflegeversicherung, Alterung, medizinischer Fortschritt

\section{Wir werden älter, kränker und teurer}

Die deutsche Bevölkerung altert zusehends. Die ferne Lebenserwartung der 65-jährigen nimmt stetig jedes Jahrzehnt um mehr als ein Jahr zu, und die Geburtenzahl liegt etwa ein Drittel niedriger, als es zur Bestandserhaltung der

1 Teile dieses Übersichtsaufsatzes basieren auf Breyer (2014). Insbesondere die Abschnitte 2.2 und 3.2 sind hier jedoch wesentlich ausführlicher.

*Kontaktperson: Friedrich Breyer, Lehrstuhl für Wirtschafts- und Sozialpolitik, Universität Konstanz, D-78457 Konstanz,

E-Mail: friedrich.breyer@uni-konstanz.de
Bevölkerung erforderlich wäre (OECD 2013). Als Folge wird der Altenquotient (also die Zahl der Personen jenseits der 65 Jahre im Verhältnis zur Zahl der Personen im Erwerbsalter von 20 bis 65 Jahren), der im Jahr 2000 noch 27 Prozent betragen hat, bis 2060 auf 65 Prozent steigen. Das ist weit mehr als das Doppelte (vgl. Abbildung 1).

Mit Blick auf die Finanzierung der Gesundheitsausgaben muss dieser Prozess aus zwei Gründen Besorgnis erregen: Zum einen steigen die Ausgaben für medizinische Behandlung im Laufe des Lebens typischerweise (vgl. Abbildung 2). Zum anderen sind die Ausgaben in der gesetzlichen Krankenversicherung (GKV) durch eine Umlage finanziert. Das bedeutet, dass die GKV keine nennenswerte Kapitalreserve hat, sondern laufende Ausgaben immer durch laufende Beitragseinnahmen deckt. Da aber die Ausgaben primär für ältere Menschen getätigt werden, die Beiträge jedoch auf Arbeitseinkommen (und Renten) erhoben und diese Einnahmen daher primär von den Erwerbstätigen aufgebracht werden, muss man an der Finanzierbarkeit zweifeln, wenn sich die Zahl der Erwerbstätigen relativ zur Zahl alter Menschen verringert. Hinzu kommt noch, dass auch der medizinische Fortschritt geeignet ist, die Gesundheitsausgaben in die Höhe zu treiben. Dabei ist der Begriff „Gesundheitsausgaben“ im umfassenden Sinn zu verstehen und beinhaltet somit auch Ausgaben für die Pflege.

\section{Zur Altersabhängigkeit der Gesundheits- und Pflegeausgaben}

\subsection{Theorie}

\subsubsection{Alterung und Ausgabenentwicklung}

Die für die Prognose der Ausgabenentwicklung entscheidende Frage lautet: Wie werden sich die in Abbildung 2 dargestellten Ausgabenprofile mit zunehmender Bevölke- 


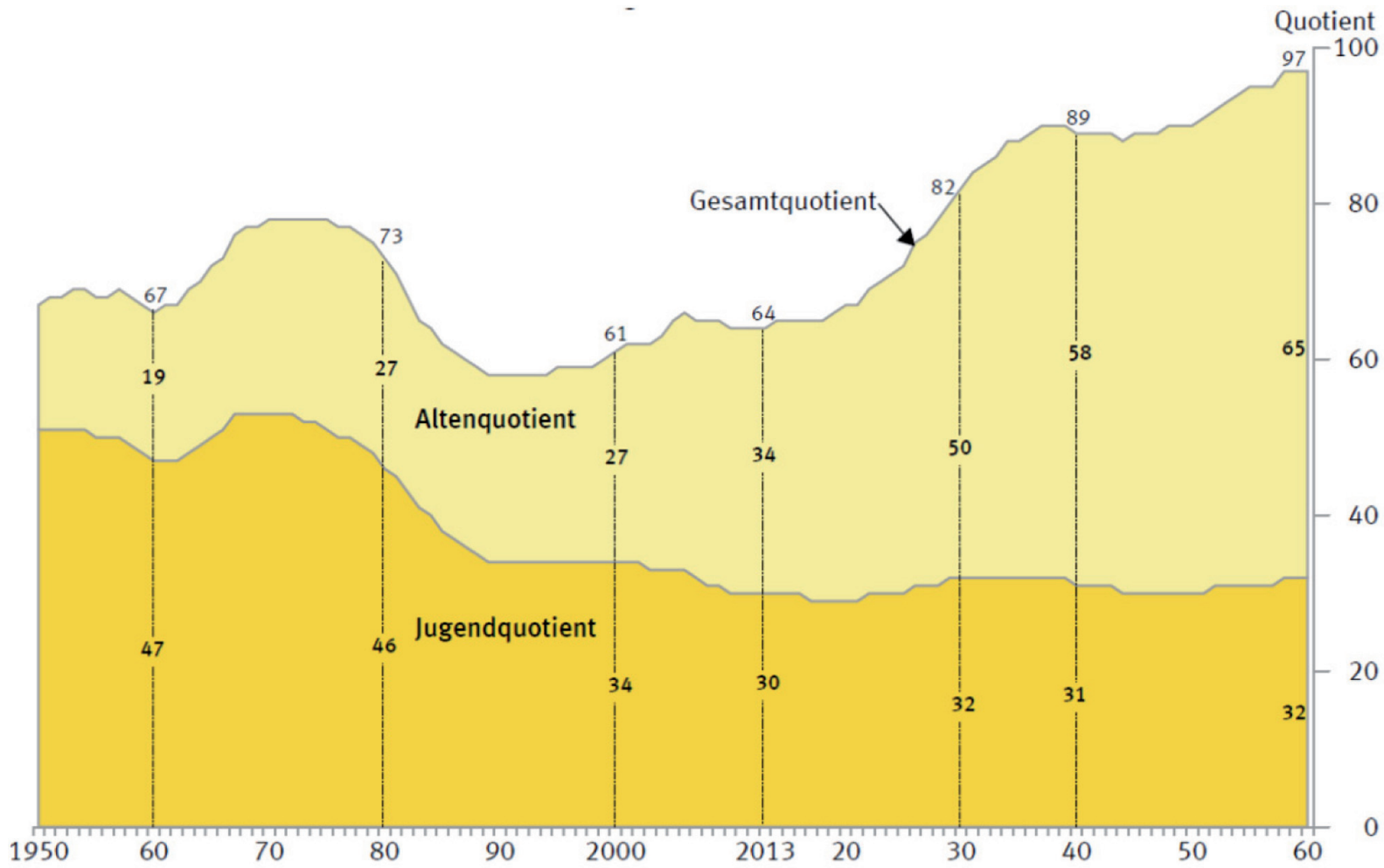

Abbildung 1: Entwicklung des Altenquotienten bis 2060.

Quelle: Statistisches Bundesamt (2015a), S. 26.

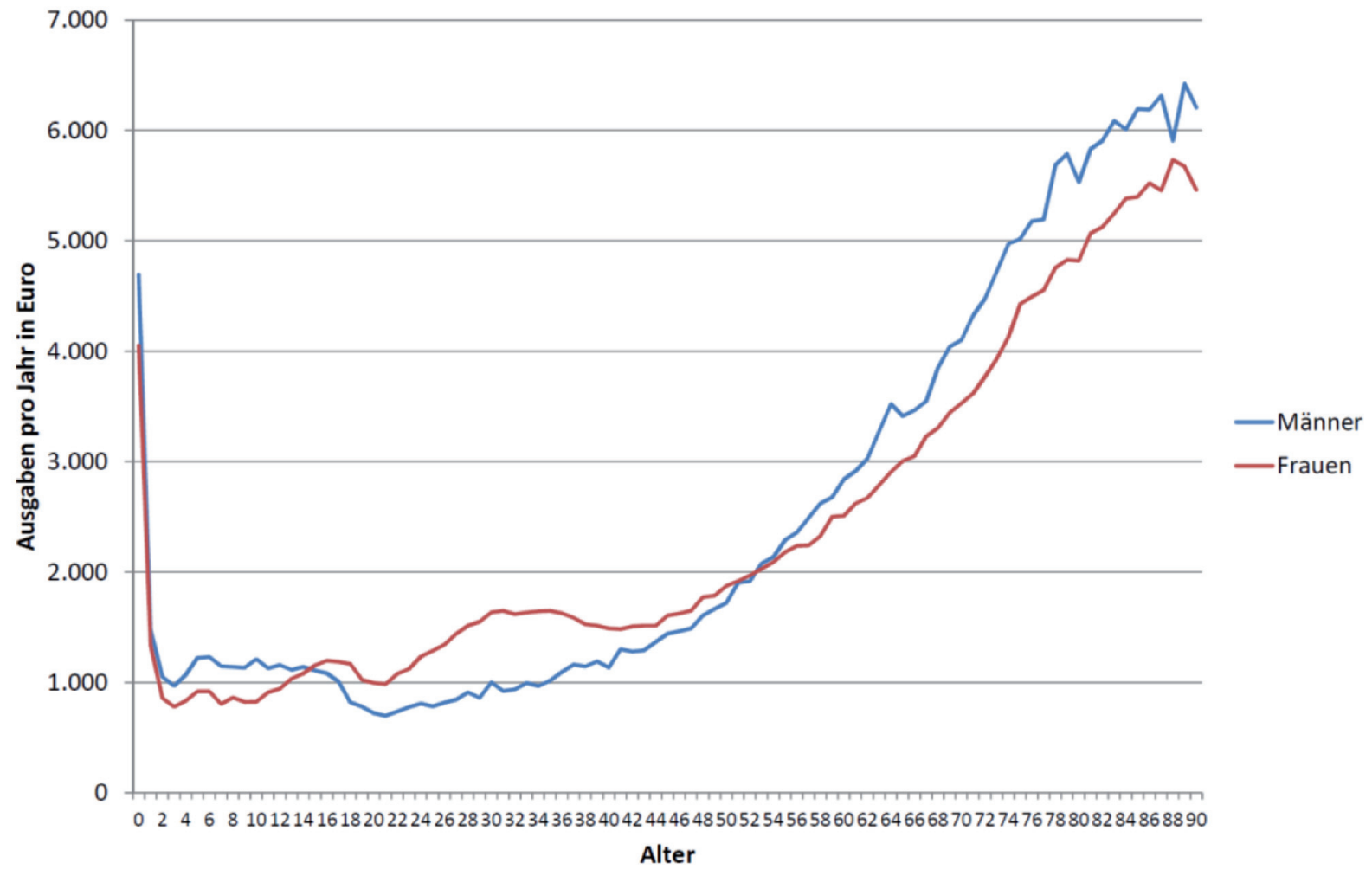

Abbildung 2: Alters-Ausgabenprofile für Frauen und Männer in der GKV 2009.

Quelle: Eigene Berechnungen aus Risikostrukturausgleichsdaten des Bundesversicherungsamts. 
rungsalterung ändern? Hierzu sind zunächst zwei grundlegende Fragen zu beantworten: (1) Hängen die Gesundheitsausgaben (1a) primär davon ab, wie krank und behandlungsbedürftig die Bevölkerung ist, sind sie also von der individuellen Nachfrage determiniert, oder spielen dafür (1b) politische Entscheidungen, also die Angebotsseite, eine entscheidende Rolle?

Falls Frage (1a) bejaht wird, ist weiter zu fragen: (2) Ist das Lebensalter gut geeignet, Krankheit und Behandlungsbedürftigkeit vorherzusagen, oder hängen diese von anderen Bestimmungsgründen ab? Auf die zweite Frage gibt es dann wiederum prinzipiell drei mögliche Antworten: (2a) Ja, aus dem Lebensalter lässt sich gut auf die Behandlungsbedürftigkeit schließen, und somit sollte das Profil der altersspezifischen Pro-Kopf-Ausgaben nur vom Stand der Medizintechnik abhängen und daher gleich bleiben, wenn man diesen gedanklich konstant hält („Status-quoHypothese").

Oder (2b): Nein, der beobachtbare Anstieg der Gesundheitsausgaben mit dem Lebensalter zeigt primär an, dass mit steigendem Alter mehr Menschen sterben und dass die letzten Jahre vor dem Tod besonders hohe Gesundheitskosten verursachen. Dies haben beispielsweise Lubitz und Riley (1993) gezeigt: Unter den mehr als 65 Jahre alten Versicherten in den Vereinigten Staaten waren die Ausgaben im letzten Lebensjahr etwa sechsmal so hoch wie durchschnittlich in einem anderen Jahr. Entscheidend für die Ausgaben eines Einzelnen ist demnach weniger der zeitliche Abstand von der Geburt als der zeitliche Abstand zum Tod. Diese Einsicht, auch „Nähe-zum-Tod-Hypothese“ (Time-to-death hypothesis) genannt, verdanken wir meines Wissens Fuchs (1984).

Und schließlich (2c): Die Frage ist falsch gestellt; der unterstellte positive Zusammenhang zwischen Krankheit und Ausgaben gilt nicht uneingeschränkt, da Krankheit keine eindimensionale Größe ist, sondern unterschiedliche Facetten hat. So werden bestimmte teure Behandlungen gerade an solchen Patienten vorgenommen, die zwar an einer schweren Krankheit leiden (beispielsweise Nierenversagen oder Verschleiß des Hüftgelenks), sich aber in einem guten Allgemeinzustand befinden (andernfalls wäre das Operationsrisiko zu hoch). Die teuersten Patienten wären danach nicht diejenigen mit dem „schlechtesten“ Gesundheitszustand.

Welche Konsequenzen ergeben sich nun aus den verschiedenen denkbaren Antworten für die weiter gehende Frage, wie sich die Gesundheitsausgaben entwickeln, wenn die Lebenserwartung steigt?

Ad (2a): Wenn das Lebensalter primär die Ausgaben bestimmt und - zum Beispiel durch medizinischen Fortschritt - mehr Menschen höhere Altersstufen errei- chen, so folgt das, was Krämer (1993) die „Medikalisierung des Alterns“ und Olshansky et al. (1991) „Expansion of morbidity" nennen. Viele ältere Patienten leiden an mehreren Krankheiten gleichzeitig, sind also „multimorbid“. Somit können neue Möglichkeiten, eine dieser Krankheiten (beispielsweise eine Herz-Kreislauf-Erkrankung) zu behandeln, das Leben des Patienten zwar verlängern, ihn aber nicht gesund machen. Vielmehr mag schon bald ein anderes Leiden (beispielsweise Krebs) wieder neue Behandlungen erfordern. Die Phase erhöhter Behandlungsbedürftigkeit vor dem Tod wird also länger und damit teurer. Im Ergebnis erhöht die Alterung die Gesundheitsausgaben pro Kopf.

Ad (2b): Wenn die Behandlungsbedürftigkeit primär mit dem zeitlichen Abstand zum Tod zusammenhängt und die Lebenserwartung - beispielsweise durch eine gesündere Lebensweise - steigt, wird die Phase der schweren und einer Behandlung bedürfenden Krankheiten und der hohen Ausgaben nach hinten verschoben, ändert sich aber nicht in ihrer Länge. Relativ zur gestiegenen Lebensdauer macht die Krankheitsphase somit einen geringeren Anteil aus, so dass die Pro-Kopf-Ausgaben durch die Alterung leicht sinken (,schwache Kompressionshypothese“).

Die „starke Kompressionshypothese“, die auf Fries (1980) zurückgeht, besagt demgegenüber, dass durch eine gesündere Lebensweise und die Zurückdrängung akuter Erkrankungen zwar sowohl die gesunde Lebenszeit als auch die mittlere Lebensdauer steigt, nicht jedoch die (maximale) Lebensdauer. Die Folge davon ist, dass die Phase hoher Ausgaben am Lebensende nicht nur relativ, sondern sogar absolut kürzer wird und sich die Alterung damit stark dämpfend auf die Pro-Kopf-Ausgaben auswirkt.

Eine ähnliche Folgerung ergibt sich auch dann, wenn man die schwache Kompressionshypothese mit der Vermutung einer Altersrationierung im Gesundheitswesen verbindet. Soll heißen: Die Ärzte schöpfen in der Behandlung von schwerkranken Patienten in sehr hohem Alter nicht mehr alle Möglichkeiten aus, anders als im Fall etwas jüngerer Patienten ${ }^{2}$. Falls sich die Phase der schweren Krankheiten ins höhere Alter verschiebt, sinken durch diesen Effekt deren Kosten und wiederum nehmen die ProKopf-Ausgaben ab.

Ad (2c): Wenn die höchsten Kosten bei Patienten mit einem guten Allgemeinzustand auftreten, könnten eine gesündere Lebensweise und die damit verbundene höhere Lebenserwartung die Ausgaben für ältere Patienten sogar

2 Die Praxis der Rationierung in sechs Ländern einschließlich Deutschlands beschreiben Strech et al. (2008). Zur ethischen Rechtfertigung der Altersrationierung vgl. Daniels (1985) und Callahan (1987). 
steigern. Denn zum einen geht das Operationsrisiko zurück und zum anderen können die Patienten von einer Behandlung (beispielsweise von einem künstlichen Hüftgelenk) aus der Sicht der Ärzte noch länger profitieren als bei geringerer Lebenserwartung. Mit anderen Worten: ein 80-jähriger würde im Jahr 2040 ähnlich intensiv behandelt werden wie ein 75 -jähriger heute, wenn beide ungefähr die gleiche verbleibende Lebenserwartung haben. Breyer et al. (2015) nennen dies den „Eubie-Blake-Effekt“ des Arztverhaltens ${ }^{3}$. Die Lebenserwartung selbst ist dann geeignet, hohe Pro-Kopf-Ausgaben vorherzusagen.

Schließlich muss noch der Fall betrachtet werden, dass Frage (1b) bejaht wird, die Gesundheitsausgaben eines Landes also nicht aus der Behandlungsbedürftigkeit der Bevölkerung durch Summation errechnet werden können, sondern politisch steuerbar sind. Unter dieser Annahme haben Zweifel und Ferrari (1992) die Hypothese vom „Sisyphus-Syndrom im Gesundheitswesen “ aufgestellt, nach der hohe Gesundheitsausgaben die Lebenserwartung steigern und damit den Anteil der Alten an der Bevölkerung und der Wählerschaft erhöhen. Ein hoher Anteil alter Wähler wirkt sich ihnen zufolge im politischen Prozess dahingehend aus, dass die öffentlichen Gesundheitsausgaben steigen, weil alte Menschen davon besonders profitieren. Im Ergebnis seien Lebenserwartung und Gesundheitsausgaben positiv miteinander korreliert. Doch die Kausalität sei nicht, wie in (2b) behauptet, einseitig, sondern die beiden Größen bedingten einander gegenseitig.

Hall und Jones (2007) haben eine ähnliche Hypothese aufgestellt, nach der das Wachstum der Lebenserwartung und der Gesundheitsausgaben letztlich auf die Zunahme der Pro-Kopf-Einkommen zurückgeht. Wenn man zusätzlich einen abnehmenden Grenznutzen des Konsums annimmt, wird mit wachsendem Einkommen eine Ausdehnung der Lebensspanne immer wichtiger. Eine solche kann man durch höhere Gesundheitsausgaben zu erzielen hoffen, die also nicht als (möglichst zu vermeidende) Kosten, sondern als (bewusst getätigte) Investitionen zu betrachten sind.

\subsubsection{Medizinischer Fortschritt und Ausgabenentwicklung}

Allgemein unterscheiden Ökonomen zwei Arten des technischen Fortschritts. Die erste Art führt zu Prozessinnova-

3 Eubie Blake war ein amerikanischer Jazz-Pianist, der an seinem (angeblichen) 100. Geburtstag sinngemäß sagte: „Wenn ich gewusst hätte, dass ich so lange lebe, hätte ich mich mehr um meine Gesundheit gekümmert.“ tionen und erlaubt es, bekannte Güter mit weniger Ressourcenaufwand herzustellen; die zweite Art führt zu Produktinnovationen und erweitert damit das verfügbare Spektrum von Gütern. Die erste Art senkt prinzipiell die Produktionskosten - man denke zum Beispiel an den Preis eines durchschnittlichen PCs in den achtziger Jahren und heute. Allerdings senkt sie nicht notwendigerweise die Ausgaben für dieses Gut, sofern die Nachfrage genügend stark (und positiv) auf die Preissenkung reagiert. Ein gutes Beispiel aus der Medizin sind die laparoskopischen („minimalinvasiven“) Operationen, etwa am Meniskus: deren Kosten sind in den vergangenen Jahrzehnten deutlich gesunken, ebenso das Operationsrisiko, so dass das Volumen in die Höhe geschnellt ist. Der Gesamteffekt auf die Ausgaben ist also ungewiss.

Klarer ist das Bild bei den Produktinnovationen, sprich: bei prinzipiell neuen Diagnose- und Therapieformen, zum Beispiel durch innovative Arzneimittel. Viele von ihnen erweitern das Therapiespektrum auf Krankheiten, die zuvor überhaupt nicht oder nur symptomatisch behandelt werden konnten. Damit sind sie eindeutig ausgabensteigernd. Das beste Beispiel hierfür liefert die Transplantationsmedizin, die vor 50 Jahren noch nicht existierte und für die heute Milliarden ausgegeben werden. Daraus folgt wiederum, dass ein Anstieg der Gesundheitsausgaben, der auf eine Verbesserung des „Produkts“ medizinische Behandlung zurückgeht, nicht negativ beurteilt werden muss, sondern schlicht den Präferenzen der Bevölkerung entspricht.

\subsection{Empirie}

Um den Einfluss der Alterung und des medizinischen Fortschritts auf die Gesundheitsausgaben empirisch zu ermitteln, bieten sich prinzipiell drei Wege an:

1) Individualdaten: Wenn man von einer großen Zahl von Individuen (Versicherten) das Alter, die Ausgaben in einem Zeitraum und das Sterbedatum kennt, kann man daraus den Zusammenhang zwischen Alter, Nähe zum Tod und Ausgaben ermitteln. Meist handelt es sich dabei allerdings um Daten nur eines Jahres, so dass der Einfluss medizinischen Fortschritts nicht Gegenstand der Untersuchung sein kann.

2) Länderdaten: Hier beziehen sich die Daten auf die Ausgaben eines oder mehrerer Länder, meistens zu verschiedenen Zeitpunkten, also als Panelerhebungen. Die Alterszusammensetzung der Bevölkerung kann man bisweilen nur grob messen, beispielsweise durch den Anteil der Personen jenseits des Alters von 65 Jahren. Der Einfluss des medizinischen Fort- 
schritts wird in der Regel am Ausgabentrend abgelesen.

3) Gruppendaten: Hier ist die Beobachtungseinheit eine nach Alter und Geschlecht homogene Gruppe innerhalb eines Landes. Im Vergleich zu Individualdaten lässt sich hier zwar nicht die Nähe zum Tod beobachten, sondern lediglich der Anteil der Gruppenmitglieder, die in der betrachteten Periode gestorben sind. Doch immerhin lässt sich die Lebenserwartung berechnen.

Schließlich kann man die Studien noch danach unterscheiden, ob sie die gesamten Gesundheitsausgaben erfassen oder nur diejenigen in bestimmten Leistungsbereichen (beispielsweise ambulante und stationäre Versorgung oder Pflege).

\subsubsection{Alter und Gesundheitsausgaben: Individualdaten}

\subsubsection{Gesamte Gesundheitsausgaben}

Zweifel et al. (1999) haben in ihrer berühmten Arbeit die Ausgaben von etwa 1000 Versicherten in der Schweiz untersucht, die zwischen 1983 und $1992 \mathrm{im}$ Alter von mehr als 65 Jahren gestorben waren, jeweils in den letzten 8 Quartalen vor deren Tod. Sie stellten fest, dass die Ausgaben in diesen 8 Quartalen signifikant stiegen, dass jedoch das Alter des Patienten keine Rolle spielte, auch dann nicht, wenn man den Zeitraum auf die letzten 5 Jahre vor dem Tod ausdehnte. Sie schlossen daraus: "Exclusive emphasis on population ageing as a cause of growth in per capita health care expenditure runs the risk of creating a red herring by distracting from the choices that ought to be made ...” (S. 494).

Dies war nicht nur eine überzeugende Bestätigung der Time-to-death-Hypothese, sondern die Geburtsstunde der hernach viel zitierten „Red-herring-Hypothese“, die einem ganzen Literaturzweig den Namen gab ${ }^{4}$. Die Autoren suggerierten, dass der Zusammenhang zwischen Alterung und Gesundheitsausgaben von Leuten in die Welt gesetzt worden sei, die von den wahren Gründen für den Ausgabenanstieg im Gesundheitswesen (nämlich Ineffizienz und Überversorgung) ablenken wollten.

Die Arbeit von Zweifel et al. (1999) wurde in der Folge mit verschiedenen Argumenten kritisiert. Schon die Autoren räumten ein, dass sie lediglich für Patienten, die wenige

4 Leser englischer Krimis wissen, dass ein „Red herring“ eine falsche Fährte ist, die ein Missetäter (oder der Autor) bewusst legt, um seine Verfolger (bzw. Leser) in die Irre zu führen.
Jahre vor dem Tod standen, gezeigt haben, dass das Lebensalter keine Rolle spielt, nicht aber für die überwiegende Mehrheit der Bevölkerung, für die das nicht gilt. Darüber hinaus wurde auch die ökonometrische Vorgehensweise bemängelt. Salas und Raftery (2001) und Seshamani und Gray (2004a) wiesen darauf hin, dass die Variable „Nähe zum Tod" nicht unbedingt exogen sein muss, sondern dass die Überlebensdauer auch von den Behandlungsausgaben abhängen kann.

Nachfolgende Arbeiten haben diese Mängel jedoch behoben und trotzdem ähnliche Resultate erbracht. Felder et al. (2000) betrachteten eine Teilmenge der Daten aus Zweifel et al. (1999) und zeigten, dass für Personen jenseits des Alters von 65 Jahren - bei gleicher Zeitspanne bis zum Tod - die Ausgaben mit höherem Alter sogar abnahmen. Zweifel et al. (2004) untersuchten wiederum Schweizer Ausgabendaten für Personen, die innerhalb der nächsten 4 Jahre gestorben waren, und Überlebende. Dabei stellten sie fest, dass der Anstieg der Ausgaben mit dem Alter jenseits des 50. Lebensjahrs in jeder dieser beiden Gruppen für sich genommen erheblich geringer ausfällt, als wenn man beide Gruppen gemeinsam betrachtet (vgl. Abbildung 3). Auch dies bestätigt die Time-to-death-Hypothese. Die Red-herring-Hypothese allerdings wird nicht gestützt, da sich insbesondere bei den Überlebenden eine Verdreifachung der jährlichen Ausgaben zwischen dem 50. und 90. Lebensjahr feststellen lässt. Felder et al. (2010) berücksichtigten dann, dass die Überlebenszeit etwas mit den Gesundheitsausgaben zu tun haben kann, und fanden heraus, dass dies nichts an der zentralen Aussage ändert, dass die Zeitspanne bis zum Tod und nicht das Alter die wichtigste Determinante der Ausgaben ist.

Interessanterweise stellten Shang und Goldman (2008) fest, dass selbst der zeitliche Abstand zum Tod in seiner Bedeutung für die Erklärung der Gesundheitsausgaben abnimmt, wenn man Maße für den Gesundheitszustand der Patienten (wie den Body-Mass-Index, das Rauchen und das Vorliegen bestimmter Erkrankungen wie Diabetes, Krebs oder Schlaganfall) in das Erklärungsmodell aufnimmt.

\subsubsection{Ausgaben in einzelnen Leistungsbereichen}

Seshamani und Gray (2004b) betrachteten ausschließlich Ausgaben für Krankenhausbehandlungen in Großbritannien und konnten zeigen, dass diese bereits 15 Jahre vor dem Tod zu steigen beginnen und mit dem Alter nur bis zum 80. Lebensjahr steigen, danach jedoch abfallen. Gregersen und Godager (2013) haben in einer ganz neuen, noch unveröffentlichten Arbeit alle Krankenhausfälle in Norwegen über den Zeitraum von 12 Jahren (1998 bis 2009) 


\section{Ausgaben in CHF}

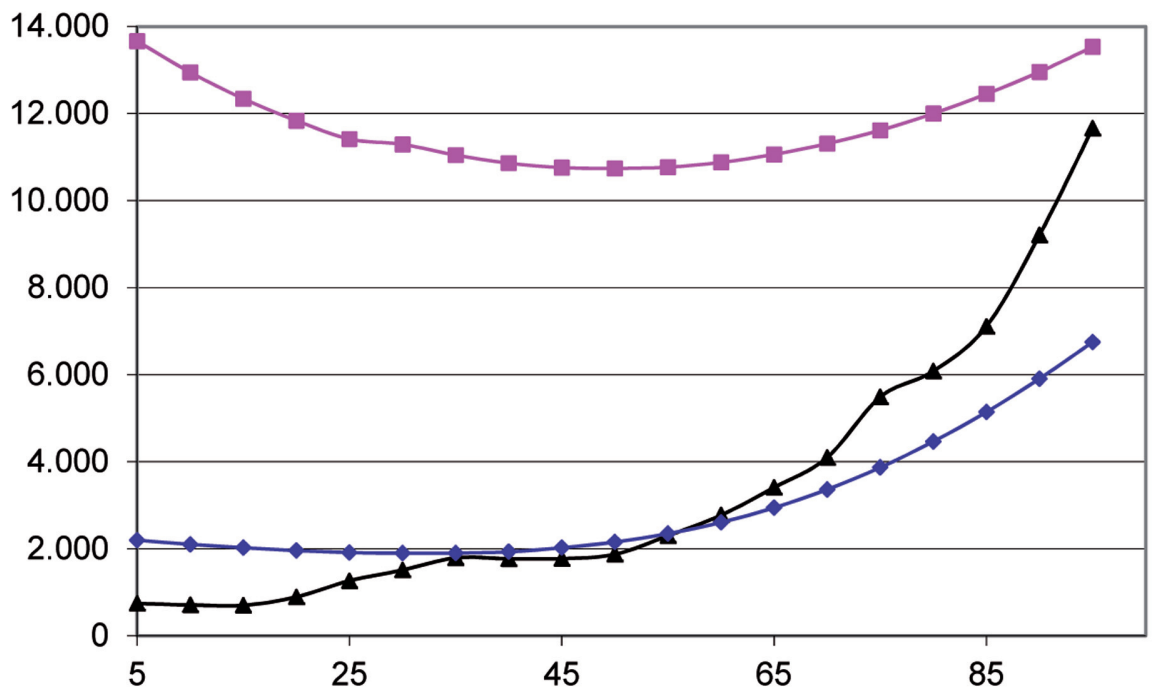

Alter

\section{$\multimap$ tats. Ausgaben $\multimap$ Ausg. Überlebende $\rightarrow$ Ausg. Verstorbene}

Abbildung 3: Tatsächliche Durchschnittsausgaben (schwarz) und geschätzte Ausgabenprofile für Überlebende (blau) und Verstorbene (rot), Schweiz 1999.

Quelle: Zweifel et al. 2004, S. 663.

untersucht und gezeigt, dass sowohl das Alter als auch die Nähe zum Tod signifikant in der Lage sind, die Ausgaben vorherzusagen. Dabei machen die Ausgaben im letzten Lebensjahr 9,2 Prozent aller Krankenhausausgaben aus und nehmen mit dem Alter des Sterbenden ab. Hingegen zeigt Gregersen (2014) am gleichen Datensatz, dass die Ausgaben (für Überlebende) nicht nur mit dem Alter steigen, sondern dass dieser Anstieg in jüngster Zeit steiler geworden ist (dass der Altersgradient der Krankenhausausgaben also mit der Zeit gestiegen ist).

Wong et al. (2011) haben das Ergebnis, dass die Nähe zum Tod die Kosten stärker treibt als das Lebensalter, auf der Grundlage von krankheitsspezifischen Krankenhausausgaben für 94 verschiedene Krankheiten in den Niederlanden bestätigt. Atella und Conti (2013) hingegen stellten fest, dass das Alter wesentlich besser geeignet ist, den Umfang ambulanter Behandlungsausgaben vorherzusagen, als die Nähe zum Tod: Während sich die Ausgaben zwischen dem 40. und dem 80. Lebensjahr verfünffachen, steigen sie kurz vor dem Tod nur um 30 Prozent.

Auch für Pflegeausgaben zeigten De Meijer et al. (2009), dass das Alter eine signifikante Rolle spielt, während die Nähe zum Tod lediglich als Proxy für den Grad der Pflegebedürftigkeit dient und insignifikant ist, wenn letzterer einbezogen wird. Ähnliches fanden Werblow et al.
(2007) anhand eines schweizerischen Datensatzes für 1999 heraus, in dem das Alter allein bei den Pflegeausgaben eine Rolle spielt, während die restlichen Ausgaben mit dem Alter eher abnehmen. Karlsson und Klohn (2014) zeigten auch für Schweden, dass die Pflegeausgaben maßgeblich vom Alter und sehr viel geringer von der Nähe zum Tod determiniert werden.

\subsection{Alter, Zeittrend und Gesundheits- ausgaben: Bevölkerungsdaten}

Eine entscheidende Schwäche der meisten im vorangegangenen Abschnitt dargestellten empirischen Analysen besteht darin, dass sie auf Querschnittsdaten beruhen, also die Auswirkungen der demographischen Alterung, vor allem in Form steigender Lebenserwartung, im Zeitverlauf nicht abbilden können ${ }^{5}$. Aufschluss über diesen Zusammenhang können nur Zeitreihendaten oder kombinierte Querschnitts-Zeitreihen-Daten geben, sogenannte PanelStudien, deren Beobachtungseinheiten nicht Individuen,

5 Ausnahmen waren Seshamani und Gray (2004b) und Felder et al. (2010). 
sondern Bevölkerungen (beispielsweise jeweils eines Landes) sind.

Breyer und Ulrich (2000) haben versucht, anhand einer Zeitreihe von Pro-Kopf-Ausgabendaten für die gesetzliche Krankenversicherung (1970 bis 1995) insgesamt den Einfluss von Alterung, Einkommenswachstum und medizinischem Fortschritt zu isolieren. Die Altersstruktur, allerdings nur grob gemessen durch den Anteil der Versicherten jenseits der 65 Jahre, erwies sich ebenso als signifikanter Erklärungsfaktor wie die Höhe des Sozialprodukts. Schließlich deutete der Zeittrend auf ein jährliches Wachstum der Gesundheitsausgaben hin, das um 1 Prozentpunkt über dem Wachstum des Sozialprodukts liegt. Studien für andere Länder (zum Beispiel Barros 1998) ergaben dagegen keinen signifikanten Einfluss des Anteils alter Menschen auf die Gesundheitsausgaben eines Landes.

Zweifel et al. (2005), die die wechselseitige Beeinflussung von Lebenserwartung und Gesundheitsausgaben untersuchten, stellten hingegen für eine Stichprobe von 30 OECD-Ländern über den Zeitraum 1970-2000 fest, dass die Variable, die zugleich die Lebenserwartung und den Anteil der Personen im Alter von mehr als 65 Jahren misst, die Gesundheitsausgaben durchaus signifikant positiv beeinflusst.

Schließlich haben Breyer et al. (2015) die Hypothese des Vorliegens eines Eubie-Blake-Effekts anhand von Daten aus dem GKV-Risikostrukturausgleich überprüft, die für jede Altersgruppe eines Jahrgangs über den Zeitraum 1997-2009 die Pro-Kopf-Ausgaben für Behandlung (Krankenhaus, Arzneimittel sowie Heil- und Hilfsmittel) getrennt nach Geschlechtern ausweisen. Für diese Gruppendaten tritt der Anteil Verstorbener (die „Sterberate“) an die Stelle des Zeitabstands zum Tod, und die Lebenserwartung wird durch den Anteil jener Personen approximiert, die mindestens noch fünf Jahre weiterlebten (die Fünf-JahresÜberlebensrate). Es zeigt sich, dass sowohl die Sterberate (im Einklang mit der Time-to-death-Hypothese) als auch die Fünf-Jahres-Überlebensrate (im Einklang mit dem Vorliegen eines Eubie-Blake-Effekts) einen signifikanten Einfluss auf die Pro-Kopf-Ausgaben einer Altersgruppe hat, dass aber, wenn man beides konstant hält, immer noch ein deutlicher Anstieg der Ausgaben mit dem Alter sowie ein positiver Zeittrend zu erkennen sind.

\subsection{Implikationen für die künftige Ausgabenentwicklung}

\subsubsection{Projektionen für die Entwicklung der Gesundheitsausgaben}

Die Verfasser sämtlicher dargestellten Arbeiten versuchen, den Zusammenhang zwischen Alter(ung) und Gesundheitsausgaben in der Vergangenheit zu ermitteln. Der eigentliche Zweck der Übung besteht jedoch darin, empirisch fundierte Voraussagen zur wahrscheinlichen künftigen Entwicklung der Gesundheits- und Pflegeausgaben zu treffen. Solche Voraussagen sind allerdings insofern problematisch, als sie politische Entscheidungen, die angesichts der Knappheit öffentlicher Mittel getroffen werden, naturgemäß nicht berücksichtigen können. Der Sinn solcher Simulationsrechnungen kann also nur darin bestehen, den „Bedarf“ an Gesundheitsausgaben in der Zukunft zu ermitteln und somit möglicherweise bestehende Lücken in den Finanzierungsmöglichkeiten früh zu erkennen.

Die wichtigste Erkenntnis aus den entsprechenden Arbeiten aus verschiedenen Ländern lautet: Wenn man die Simulationen auf empirischen Schätzungen der Gesundheitsausgaben aufbaut, bei denen die Variable „Zeit bis zum Tod“ einbezogen ist, dann ergibt sich für die Zukunft ein weniger dramatischer Anstieg der Ausgaben als in Schätzungen, in denen dieser Effekt keine Berücksichtigung findet. Erstmals haben dies Miller (2001) sowie Stearns und Norton (2004) für die Vereinigten Staaten gezeigt. Letztere verglichen Hochrechnungen für die Medicare-Ausgaben des Jahres 2020 aus Ausgabendaten für den Zeitraum 1992-1998 und stellten fest, dass die Nichtberücksichtigung des Time-to-death-Effekts zu einer Überschätzung der Gesamtausgaben eines durchschnittlichen Medicare-Versicherten (vom 65. Lebensjahr an) um bis zu 15 Prozent (nämlich auf 117.000 statt 102.000 Dollar) führt.

Wenn man diese Überschätzung zum tatsächlichen Anstieg über diesen Zeitraum in Beziehung setzen will, muss man die Höhe der Ausgaben in den neunziger Jahren kennen. Nach Lubitz et al. (1995) betrugen sie im Jahr 1990 etwa 53.000 Dollar. Der „korrekt“ geschätzte Anstieg über diesen Zeitraum beträgt daher etwa 92 Prozent, der „überschätzte“ 121 Prozent. Das Ausmaß der Überschätzung beträgt mithin ein knappes Drittel des „tatsächlichen“ Anstiegs. Polder et al. (2006) fanden für die Niederlande lediglich eine Überschätzung der Wachstumsrate der Gesundheitsausgaben durch das „falsche“ Modell von 10 Prozent, Geue et al. (2014) für Krankenhausausgaben in Schottland eine Überschätzung von etwa 17 Prozent. Einen wesentlich größeren Überschätzungseffekt von etwa 
50 Prozent stellten Bjørner und Arnberg (2012) für Dänemark fest, während Van Baal und Wong (2012) für die Niederlande sowie Colombier und Weber (2011) für die Schweiz errechneten, dass die Sterbekosten für langfristige Projektionen der Gesundheitsausgaben überhaupt keine Rolle spielen.

Breyer und Felder (2006) verwendeten das auf Schweizer Daten beruhende Schätzmodell aus Zweifel et al. (2004) für eine Simulation der Ausgabenentwicklung im Zeitraum 2002-2050 für Deutschland auf der Basis der Bevölkerungsprognosen des Statistischen Bundesamts. Gegenüber einer Status-quo-Projektion auf der Grundlage des Alters-Ausgaben-Profils von 2002, die einen rein demographisch bedingten Anstieg der Pro-Kopf-Ausgaben bis 2050 um 24 Prozent ergibt, reduziert sich dieser $\mathrm{Zu}$ wachs durch explizite Unterscheidung der Ausgaben für Überlebende und Sterbende auf 19,5 Prozent sowie durch die Annahme der Gültigkeit der schwachen Kompressionshypothese auf 14 Prozent. Die überraschende Erkenntnis aus diesen Simulationen ist also, dass auch bei Berücksichtigung aller dämpfenden Faktoren immer noch ein positiver demographischer Effekt auf die Gesundheitsausgaben verbleibt, was auch Steinmann et al. (2007) für die Schweiz bestätigten.

Auf der anderen Seite verblasst die Höhe des demographischen Effekts gegenüber den Auswirkungen des medizinischen Fortschritts auf die Gesundheitsausgaben. Wenn man für diese gemäß der Schätzergebnisse aus Breyer und Ulrich (2000) eine jährliche Wachstumsrate von einem Prozent (über das Wachstum des Sozialprodukts hinaus) ansetzt, steigen die Pro-Kopf-Ausgaben in Deutschland zwischen 2002 und 2050 inflationsbereinigt um 101 Prozent, wovon der demographisch bedingte Anstieg nur einen kleinen Teil ausmacht. In der gleichen Weise kamen Dormont et al. (2006) für Frankreich zu dem Ergebnis, dass der Einfluss des medizinischen Fortschritts auf das Wachstum der Gesundheitsausgaben 3,8 mal so groß ist wie der Einfluss der Alterung der Bevölkerung.

Schließlich haben auch Breyer et al. (2015) eine Simulation der Pro-Kopf-Ausgaben für GKV-Mitglieder für den Zeitraum 2009-2060 auf der Basis der vorliegenden Bevölkerungsprognosen unternommen. Je nach verwendetem Schätzmodell ergibt sich daraus eine rein demographisch bedingte Entwicklung der Pro-Kopf-Ausgaben mit einer jährlichen Wachstumsrate von 0,5-1 Prozent zuzüglich eines (als medizinischen Fortschritt interpretierbaren) Zeittrends von jährlich 1-1,5 Prozent in den Ausgaben für Frauen und 2 Prozent für Männer. Auch hier zeigt sich also, dass der demographische Effekt auf die Ausgabenentwicklung zwar keineswegs null (oder gar negativ) ist, jedoch deutlich geringer bleibt als der Einfluss des medizinischen Fortschritts.

Was könnten diese Zahlen für die Entwicklung der Gesundheitsausgaben als Anteil am Bruttoinlandsprodukt (BIP) bzw. des Beitragssatzes in der GKV im Zeitraum bis 2060 nun bedeuten? Hierzu seien die Zahlen der 12. Koordinierten Bevölkerungsvorausberechnung des Statistischen Bundesamtes (2009) herangezogen, und zwar die Obergrenze der mittleren Bevölkerung. Danach werden im Jahr 2060 in Deutschland 70 Millionen Menschen leben, davon 36 Millionen im Erwerbsalter. Gegenwärtig sind es (nach Zahlen von 2014) 81 bzw. 50 Millionen. Damit schrumpft die Bevölkerung mit einer durchschnittlichen Jahresrate von 0,3 Prozent und das Erwerbspersonenpotential sogar um 0,7 Prozent. Wenn man ein langfristiges reales Wachstum der Arbeitsproduktivität von 1,5 Prozent voraussetzt, folgt daraus ein BIP-Wachstum von jährlich 0,8 Prozent, wonach das BIP im Jahr 2060 real um 44,3 Prozent höher liegen wird als 2014. Ein Trendwachstum der Gesundheitsausgaben pro Kopf von jährlich 2,3 Prozent (gemäß Breyer et al. 2015, gemittelt aus den Werten für Frauen und Männer) ergibt unter Berücksichtigung des Bevölkerungsrückgangs von jährlich 0,3 Prozent einen Gesamtanstieg der Gesundheitsausgaben bis 2060 von 148,7 Prozent, so dass der Anteil der Gesundheitsausgaben am BIP um 72,4 Prozent steigt. Wenn man ferner davon ausgeht, dass die Bemessungsgrundlage der GKVBeiträge relativ zum BIP gleich bleibt, so müsste der Beitragssatz von heute 15,5 auf 26,7 Prozent 2060 steigen.

\subsubsection{Prognosen für die Entwicklung in der Sozialen Pflegeversicherung}

Wenn man die Entwicklung der Ausgaben in der Sozialen Pflegeversicherung (SPV) im Voraus abschätzen will, muss man die folgenden Faktoren berücksichtigen:

1) die demographiebedingte Entwicklung der Pflegebedürftigkeit,

2) mögliche Anpassungen des Pflegebedürftigkeitsbegriffs (Stichwort Demenz etc.),

3) die Zusammensetzung der Inanspruchnahme von Leistungen (Geldleistung und Sachleistung; häusliche und stationäre Pflege),

4) Änderungen der Preise für Sachleistungen aufgrund veränderter Knappheitsverhältnisse auf dem Markt für professionelle Pflegekräfte.

Ad 1): Hierzu liegen Simulationsrechnungen für Deutschland von Häcker et al. (2011) sowie von Comas-Herrera et al. (2006) vor. In beiden Arbeiten gingen die Verfasser 
gemäß der Status-quo-Hypothese von konstanten altersspezifischen Pflegebedürftigkeitsraten aus und erwarteten auf dieser Grundlage von 2010 bis 2050 schon fast eine Verdopplung der Zahl der Pflegefälle in Deutschland (von 2,3 auf 4,4 Millionen). Dementsprechend prognostizierten Häcker et al. (2011) in ihrem Basismodell für das Jahr 2060 einen Beitragssatz von 5 Prozent und Comas-Herrera et al. (2006) für das Jahr 2050 einen Anteil der Pflegeversicherungsausgaben am BIP von 3,32 Prozent (gegenüber 1,24 Prozent im Jahr 2000).

Wenn man im Unterschied hierzu davon ausginge, dass Pflegebedürftigkeit in aller Regel eine Folge von Krankheit ist, wäre es plausibel, die schwache Kompressionsthese für zutreffend $\mathrm{zu}$ halten und zu erwarten, dass sich der Eintritt in die Pflegebedürftigkeit mit dem Anstieg der Lebenserwartung nach hinten verschiebt. Damit würden sich die genannten Anstiege abschwächen. Die tatsächliche Entwicklung der Pflegebedürftigkeit in Deutschland zwischen 1999 und 2011 weist allerdings nahezu konstante Pflegequoten aus (Statistisches Bundesamt, div. Jahrgänge), so dass es vernünftig erscheint, die Statusquo-Hypothese zugrunde zu legen.

Ad 2) In entgegengesetzter Richtung würde sich dagegen die vom „Beirat zur Überprüfung des Pflegebedürftigkeitsbegriffs" vorgeschlagene Ausweitung des Leistungskatalogs auswirken. Für sich genommen würde diese die Ausgaben der SPV um etwa 18 Prozent steigern, was gemäß Häcker et al. (2011) einen weiteren Beitragssatzpunkt ausmachte.

Ad 3) Eine ähnliche Wirkung hätte die Fortsetzung eines Trends, der bereits eingesetzt hat, und zwar die Umschichtung von Geldleistungen zu den (erheblich teureren) Sachleistungen in Folge einer Veränderung der Haushaltsstruktur und der Erwerbsbeteiligung von Frauen. Der zunehmende Anteil von Single-Haushalten, die verringerte Kinderzahl, die erhöhte Mobilität sowie die gestiegene Erwerbstätigkeit (und das steigende Renteneintrittsalter) von Frauen bringen es mit sich, dass immer weniger Pflegebedürftige von den eigenen Angehörigen zu Hause gepflegt werden. Dies führt zu einem höheren Anteil professioneller häuslicher Pflege und steigert die Inanspruchnahme von Heimpflege.

Ad 4) Pflegeleistungen sind vor allem persönliche Dienstleistungen, bei denen keine nennenswerten Rationalisierungspotentiale bestehen. Gerade die persönliche Hinwendung der Pflegeperson zum Patienten macht die Qualität der Leistung aus. Dies allein sorgt bereits dafür, dass der relative Preis einer Stunde Pflege zu einem industriell hergestellten Gut stetig steigen wird („Baumolsche Kostenkrankheit“, vgl. Rothgang 2011, S. 659). Zusätzlich könnte die absolut höhere Nachfrage zu einer Knappheit auf dem Markt für Pflegekräfte führen, die nur mit einer (weiteren) Lohnsteigerung bewältigt werden kann. In diesem Zusammenhang nimmt die weitere Zunahme der Erwerbsbeteiligung von Frauen, die für die Zukunft prognostiziert wird, eine ambivalente Rolle ein: Einerseits steigt dadurch auch das potentielle Angebot von Pflegekräften, andererseits fallen - sofern diese Entwicklung nicht durch pflegende Männer kompensiert wird - familiäre Pflegepersonen aus, so dass auch die Nachfrage nach professioneller Pflege zunimmt.

\subsection{Zwischenfazit zum Reformbedarf in der deutschen Kranken- und Pflegeversicherung}

Im Jahr 2015 beträgt der Gesamtbeitragssatz zur Sozialversicherung 39,55 Prozent (für Kinderlose 39,8 Prozent). Wenn man die oben errechneten Größen (26,7 Prozent für die GKV und 5 Prozent für die SPV) zu den 27,2 Prozent hinzu addiert, die Werding (2013, S. 10) in seinem „Referenzszenario" für die GRV errechnet, ergeben sich für das Jahr 2060 bereits ohne Arbeitslosenversicherung knapp 59 Prozent. Selbst bei optimistischen Annahmen über die künftige Arbeitsmarktentwicklung dürfte allein durch Such-Arbeitslosigkeit ein Beitragssatz von etwa 2 Prozent für die Arbeitslosenversicherung (ALV) hinzukommen. Lange bevor der Gesamtbeitrag auf den 61 Prozent gestiegen ist, dürften sich erhebliche politische Widerstände bemerkbar machen - ungeachtet der Tatsache, dass der Ausgabenanstieg auch eine Verbesserung der Produktqualität durch medizinischen Fortschritt widerspiegelt. Im vergangenen Jahrzehnt galt die Marke von 40 Prozent bereits als kritische Grenze, obwohl es natürlich auch damals schon medizinischen Fortschritt gab. Daraus folgt, dass jede drohende Erhöhung des Beitragssatzes in der GKV - die seit Einführung des Gesundheitsfonds eine Entscheidung des Bundestags voraussetzt und nicht mehr auf dezentralen Entscheidungen von mehr als 100 Krankenkassen beruht den Ruf nach Gegenmaßnahmen hervorbringen wird.

\section{Reformoptionen}

Welche Optionen stehen zur Verfügung, um die Kranken- und Pflegeversicherung nachhaltig zu finanzieren? In dem folgenden Versuch einer Beantwortung dieser Frage

6 Hierbei wird angenommen, dass sich der gesamte Beitragssatz zur GKV gegenüber 2014 nicht verändert. 
bleiben mögliche Reformmaßnahmen außerhalb der entsprechenden Zweige der Sozialversicherung bewusst ausgeklammert, beispielsweise die Erleichterung von $\mathrm{Zu}$ wanderung. $\mathrm{Ob}$ und unter welchen Voraussetzungen eine Zuwanderung die Finanzierungsprobleme im Gesundheitswesen mildern kann, ist in der Literatur umstritten und könnte Gegenstand eines eigenen Übersichtsaufsatzes sein.

\subsection{Reformoptionen in der Gesetzlichen Krankenversicherung}

\subsubsection{Verbesserte Einnahmen durch Bürgerversicherung oder Kopfpauschale}

Häufig ist zu hören ${ }^{7}$, dass in Zeiten sinkender Lohnquote und wachsender Lohnspreizung eine nachhaltige Finanzierung der Sozialversicherung auch dann gefährdet sei, wenn deren Ausgaben sich proportional zum Sozialprodukt verhielten. Dies liege daran, dass sich Beiträge ausschließlich nach den Arbeitseinkommen (bis zur Bemessungsgrenze) und den Lohnersatzeinkommen richteten und dass Beamte, Selbstständige und Bestverdiener von der Mitgliedschaftspflicht in GKV und SPV befreit seien. Als Abhilfe wird 1) die Einführung einer Bürgerversicherung mit Einbeziehung weiterer Einkommensarten in die Beitragserhebung oder 2) die Umstellung der Beiträge auf Kopfpauschalen mit steuerfinanziertem Sozialausgleich empfohlen.

Ad 1): Aus verfassungsrechtlichen Gründen kann sich die Einführung einer generellen Pflicht zur Mitgliedschaft in GKV und SPV nicht auf Bestandskunden der PKV (Private Krankenversicherungen) beziehen; somit ließe sich eine echte „Bürgerversicherung“ erst nach einer Übergangszeit von vielen Jahrzehnten realisieren. Wenn man weitere Bevölkerungskreise und weitere Einkommensarten einbezöge, wäre die Auswirkung auf den Beitragssatz auf wenige Zehntel Prozentpunkte begrenzt, solange die Beitragsbemessungsgrenze nicht angehoben wird (Greß und Bieback 2013, S. 26). In Anbetracht der Größenordnung des prognostizierten Beitragssatzanstiegs würde diese Einmalmaßnahme daher nur einen sehr kleinen Beitrag zur Problemlösung liefern.

Ad 2): Ähnlich sähe es für eine Umstellung der Beiträge auf Kopfpauschalen aus: Auch hier besteht der Hauptunterschied auf der Einnahmeseite von Staat und Krankenkassen darin, dass Personen mit geringen beitrags-

7 Vgl. Rürup-Kommission (BMGS 2003, S.143). pflichtigen Einnahmen, aber nennenswertem Nicht-Arbeitseinkommen (beispielsweise Rentner) dann mehr beitragen müssten als bisher, ohne einen Anspruch auf Sozialausgleich zu haben. Der Übergang hierzu wäre zwar rechtlich einfacher als zu einer Bürgerversicherung, aber wegen des Sozialausgleichs administrativ komplizierter, da die Finanzämter dazu die Einkünfte eines größeren Personenkreises von Geringverdienern erfassen müssten, die derzeit nicht einkommensteuerpflichtig sind ${ }^{8}$.

\subsubsection{Mehr Effizienz durch Stärkung des Wettbewerbs}

Während die bislang erörterten Maßnahmen jeweils eine einmalige Wirkung auf das Niveau der Einnahmen von GKV und SPV hätten, könnte eine Lösung des Problems auch darin bestehen, das Niveau der Ausgaben - wenngleich nicht den Zeittrend - durch eine Stärkung des Wettbewerbs zwischen den Krankenkassen zu senken. Dazu müsste der Gesetzgeber zwei Voraussetzungen erfüllen:

1) Zum einen müsste der Preiswettbewerb zwischen den Kassen in Gang gesetzt werden, und zwar durch eine knappere Bemessung des allgemeinen Beitragssatzes und den damit einhergehenden Zwang, Zusatzbeiträge zu erheben. Krankenkassen als nicht-gewinnorientierte Körperschaften haben nur dann einen Anreiz zur Kostensenkung, wenn sie in die Verlustzone zu sinken drohen, und Versicherte reagieren erwiesenermaßen erheblich empfindlicher auf Zusatzbeiträge als auf die vage Aussicht einer Rückzahlung durch die Kasse (Schmitz und Ziebarth 2011).

2) Zum anderen müssten den Krankenkassen wesentlich mehr Elemente der Vertragsfreiheit in die Hand gegeben werden, mit denen sich ein Wettbewerb zwischen den Leistungsanbietern herbeiführen oder intensivieren ließe. Insbesondere müsste der Kontrahierungszwang im Bereich der stationären Versorgung aufgehoben werden. So könnten die Überversorgung mit Krankenhausbetten in Ballungsgebieten und die ineffiziente Struktur mit zu vielen kleinen und wenig spezialisierten Krankenhäusern in anderen Regionen bereinigt werden (vgl. Breyer 2013).

8 Hierbei soll nicht verkannt werden, dass die Einführung von Kopfpauschalen andere Vorteile hätte wie die Stärkung des Wettbewerbs zwischen Krankenkassen und die Finanzierung der Umverteilung aus Steuern statt aus Sozialabgaben. Dadurch verbesserte sich jedoch primär die Gerechtigkeit der Finanzierung und weniger die Nachhaltigkeit. Vgl. hierzu Breyer (2012). 


\subsubsection{Verstärkte Kapitaldeckung}

Analog zur Riester-Reform in der Rentenversicherung könnte auch in der Krankenversicherung erwogen werden, dem prognostizierten Beitragsanstieg (sei er demographisch oder durch medizinischen Fortschritt bedingt) mit mehr Kapitaldeckung zu begegnen (vgl. beispielsweise Cassel und Oberdieck 2002). Selbst wenn man diese Idee prinzipiell bejaht, bedeutet dies noch nicht, dass die Bildung einer Kapitalreserve bei den Krankenkassen zu erfolgen hat. Dem steht eine Reihe von Bedenken entgegen:

1) Wenn der Kapitalbestand bei Krankenkassen läge, müsste genau geregelt werden, wann und für welche Zwecke diese Reserve eingesetzt werden darf. Auf jeden Fall müssen die Beitragszahler, aus deren überschüssigen Beiträgen die Reserve gebildet wird und für deren Leistungsbedarf im Alter sie dienen soll, davor geschützt werden, dass sie vorzeitig abgeschmolzen wird, zum Beispiel um einen politisch nicht erwünschten Beitragssatzanstieg in Zeiten der Rezession zu vermeiden. Der Verhinderung einer politisch induzierten Zweckentfremdung dient bei der Riester-Rente die Tatsache, dass es sich hier rechtlich um private Ersparnisse und nicht um Rücklagen einer öffentlich-rechtlichen Versicherung handelt.

2) Weiterhin wäre zu regeln, wie mit den dann notwendigen Alterungsrückstellungen bei Kassenwechsel umgegangen werden soll. Dies ist keineswegs ein triviales Problem, das selbst in der PKV, in der die mangelnde sogenannte Portabilität jahrzehntelang den Wettbewerb zwischen den Versicherern behindert hat, immer noch nicht zufriedenstellend gelöst ist.

\subsubsection{Explizite Rationierung}

Wie soll man nun damit umgehen, dass die Schere immer weiter aufgeht zwischen dem medizinischen Leistungsumfang, der nach dem Stand des Wissens möglich und für den Patienten nützlich wäre, und jenem Leistungsumfang, der aus einem vorgegebenen, politisch bestimmten Beitragssatz finanzierbar ist? Nach der derzeit vorherrschenden Strategie der „impliziten Rationierung“ verspricht der Gesetzgeber einerseits dem gesetzlich Versicherten eine umfassende Versorgung nach dem Stand der medizinischen Technik. Andererseits zwingt er die Ärzte de facto durch eine Vielzahl von Regulierungen zur Ausgabenbegrenzung (Deckelung der ärztlichen Gesamtvergütung, Richtwerte für Verordnungen, Wirtschaftlichkeitsprüfungen sowie im Krankenhaus ein festes Jahresbudget), Verteilungsentscheidungen bezüglich ihrer knappen Res- sourcen zu treffen. Diese Praxis ist in einem Rechtsstaat problematisch, in dem der Staat durch Ausübung von Zwang in die individuellen Entscheidungen eingreift, die Ansprüche, die er schafft, jedoch undefiniert lässt, so dass sie einer gerichtlichen Überprüfung nicht offen stehen.

Daher wäre es wünschenswert, den Leistungskatalog stärker zu operationalisieren und damit für mehr Transparenz bei Versicherten, Leistungserbringern und Krankenkassen zu sorgen. Allerdings ist der Übergang von impliziter zu expliziter Rationierung in Deutschland durch das „Nikolaus-Urteil“ des Bundesverfassungsgerichts vom 6. Dezember 2005 erheblich erschwert. Danach müssen Krankenkassen für Versicherte, die an einer lebensbedrohlichen Krankheit leiden, auch die Kosten wissenschaftlich nicht anerkannter Behandlungsmethoden übernehmen, „wenn eine nicht ganz entfernt liegende Aussicht auf Heilung oder Besserung“ besteht. Das bedeutet, dass eine explizite Eingrenzung des Leistungskatalogs der GKV beispielsweise nach den Kriterien der evidenzbasierten Medizin und der Kosteneffektivität von den Verfassungsrichtern als nicht mit den Grundrechten vereinbar angesehen und möglicherweise verboten würde.

Dennoch wird es in der Zukunft unumgänglich sein, den Leistungskatalog nach dem Kriterium der Kosten-Effektivität von Behandlungsformen einzugrenzen, wie es in Großbritannien seit langem erfolgreich praktiziert wird. Auf diese Weise lässt sich Rechtssicherheit für die Versicherten herstellen und man ermöglicht es ihnen, nicht versicherte Leistungen auf eigene Kosten oder mit Hilfe von Zusatzversicherungen zu erhalten.

\subsection{Reformoptionen in der Sozialen Pflegeversicherung}

\subsubsection{Ausbau der umlagefinanzierten Leistungen}

Eine Besonderheit der Sozialen Pflegeversicherung in Deutschland besteht darin, dass sie nur eine Teilabdeckung des Pflegekostenrisikos vorsieht. Dabei ist der abgedeckte Anteil aufgrund der bis 2008 noch fehlenden Dynamisierung seit ihrer Einführung sogar noch gesunken und beträgt gegenwärtig weniger als 50 Prozent. Kosten, die nicht von der SPV übernommen werden, müssen der Pflegebedürftige und seine Familie zunächst privat tragen. Es ist aber möglich, dass sie aufgrund dieser Belastung Ansprüche auf Hilfe zur Pflege nach SGB XII haben, einer steuerfinanzierten Sozialleistung.

Wenn die Soziale Pflegeversicherung weiterhin eine Teilkasko-Versicherung bleibt und auch in Zukunft nur die Hälfte der Pflegekosten abdeckt, wenn gleichzeitig aber 
das Rentenniveau durch den Nachhaltigkeitsfaktor immer weiter sinkt, dann werden immer mehr Pflegebedürftige die zweite Hälfte der Pflegekosten nicht mehr aus ihrem Einkommen oder ihren Ersparnissen bestreiten können. Sie werden vielmehr der Sozialhilfe bedürfen und damit der Allgemeinheit zur Last fallen, wie es schon vor Einführung der sozialen Pflegeversicherung der Fall war. Schon heute betragen die Ausgaben der Kommunen für „Hilfe zur Pflege“ jährlich knapp 4 Milliarden Euro (Statistisches Bundesamt 2015b). Der ursprüngliche Zweck der Sozialen Pflegeversicherung, eine Entlastung der Kommunen von Sozialhilfeverpflichtungen, würde damit wieder verfehlt.

Aus diesem Grund ist es nicht überraschend, dass unter den Reformvorstellungen für die SPV auch der Vorschlag zu finden ist, dass diese umlagefinanzierte Leistung in Zukunft ausgebaut werden sollte (Rothgang 2011), zum einen durch die Einbeziehung der bisher privat Versicherten in eine „Pflege-Bürgerversicherung“, zum anderen durch eine regelmäßige Dynamisierung der Leistungen. Da die Pflege eine arbeitsintensive Tätigkeit ist, bei der nur ein geringer technischer Fortschritt zu erwarten ist, käme es durch eine solche Dynamisierung jedoch zu einem stetigen Anstieg des Beitragssatzes. Die Nachhaltigkeit der Finanzierung würde also nicht gebessert, sondern verschlechtert. Einziger Vorteil dieser Option wäre die Entlastung der steuerfinanzierten Sozialhilfe im Vergleich zum Status quo.

\subsubsection{Verstärkte Kapitaldeckung}

\subsubsection{Kollektive Kapitaldeckung: Der Pflege-Vorsorgefonds}

Die Bundesregierung hat auf die Prognosen eines drastisch steigenden Beitragssatzes zur umlagefinanzierten Pflegeversicherung reagiert und beschlossen, die SPV zukunftsfest(er) zu machen. Das dafür gewählte Instrument ist ein Vorsorgefonds, in den von 2015 an 0,1 Beitragspunkte, also etwa 1,2 Milliarden Euro im Jahr, fließen sollen. Er soll von 2034 an dazu verwendet werden, den Beitragsanstieg zu bremsen.

So sehr das Ziel zu loben ist, so ungeeignet ist das Mittel, und das gleich aus drei Gründen: Erstens sind die dafür vorgesehenen Beträge viel zu gering, um etwas zu bewirken. Wenn man realistisch von einem Realzins von null ausgeht, stehen von 2034 an jährlich zusätzliche 1,2 Milliarden Euro zur Abdeckung von Pflegeleistungen zur Verfügung. Das ist bei Gesamtausgaben von 23 Milliarden Euro (2012) ein Tropfen auf den heißen Stein. Zweitens fehlen bei einem vom Staat verwalteten Fonds die Sicherungen, dass das Geld nicht früher ausgegeben wird, bei- spielsweise um in einer Rezession einen Anstieg des Beitragssatzes zu verhindern. Diese beiden Kritikpunkte wurden in der politischen Debatte bereits verschiedentlich vorgebracht.

Der dritte Kritikpunkt ist weniger bekannt, aber ökonomisch noch bedeutender: Ein Fonds, der über 20 Jahre angespart und danach aufgelöst wird, wäre adäquat, wenn es sich beim demographischen Wandel um ein vorübergehendes Phänomen handeln würde. Das ist er aber nicht. Dieses Missverständnis zeigt sich unter anderem darin, dass bei vielen Kommentatoren die Meinung vorherrscht, die Finanzierungsprobleme von Rente und Pflege in Deutschland hätten damit zu tun, dass nunmehr die geburtenstarken Jahrgänge der sechziger Jahre altern und ungefähr vom Jahr 2030 an die Renten- und Pflegekassen belasten werden. Plakativ ist vom „Altern der Baby-Boomer" die Rede. Es wird damit der Eindruck erzeugt, die Probleme seien dadurch verursacht, dass eine Generation (die der zwischen 1955 und 1975 Geborenen) besonders zahlreich sei.

In Wahrheit kommt es jedoch bei der Finanzierung von Sozialleistungen im Umlageverfahren, das auch „Generationenvertrag“ heißt, auf die absolute Größe einer Generation gar nicht an. Schon gar nicht kommt es auf ihre relative Größe gegenüber der Generation ihrer Eltern an, auf die der Begriff „Baby Boom“ hinweist. Stattdessen kommt es ausschließlich auf ihre relative Größe im Vergleich mit den nachfolgenden Generationen an, also in diesem Fall den nach 1975 Geborenen, die im Umlageverfahren die Leistungen bezahlen müssen, welche die „große Generation" erhält. Und da ist festzustellen, dass wegen der niedrigen Geburtenzahlen seit 1975 jede Folgegeneration etwa ein Drittel kleiner ist als die der jeweiligen Eltern. Nicht der „Baby Boom“ der Periode 1955-75 erschwert in Zukunft die Finanzierung der Sozialleistungen, sondern vielmehr der „Baby Bust“ seit 1975.

Letzterer ist aber augenscheinlich kein kurzzeitiger Einbruch der Geburtenziffern, sondern er setzt sich bis heute fort. Ein Ende ist trotz enormer familienpolitischer Anstrengungen verschiedener Bundesregierungen noch lange nicht abzusehen. Es wird daher nach der für die Auflösung des Vorsorgefonds vorgesehenen Periode (2034-53) keineswegs eine Entspannung der demographischen Schieflage geben, sondern diese wird sich weiter fortsetzen, wenn die Geburtenzahl nicht sehr bald und nennenswert steigt. Gesucht ist also kein einmaliges, sondern ein dauerhaftes Mittel gegen die Überlastung der Beitragszahler.

Wenn ein staatlicher Vorsorgefonds überhaupt einen Ausgleich für die fehlenden Geburten herstellen soll, müsste er kohortenweise gestaffelt werden, wobei jede Kohorte 
durch einen Geburtsjahrgang gebildet werden könnte, und die Einzahlungen einer Kohorte dürften nur dieser Kohorte im Alter zugutekommen. Da jedoch weder die Beiträge noch die Leistungen in der SPV einen Kohortenbezug haben, wäre eine solche Lösung administrativ kaum zu verwirklichen.

\subsubsection{Private kapitalgedeckte Zusatzvorsorge}

Selbst eine Dynamisierung und Leistungsausweitung kann nichts daran ändern, dass die umlagefinanzierte SPV nur eine Teilabsicherung des Pflegerisikos bieten kann. So beträgt die Leistung bei stationärer Pflege in Pflegestufe 3 seit dem 1.1.2015 monatlich 1.612 Euro, während sich die tatsächlichen Kosten des Aufenthalts in einem Pflegeheim bereits heute zwischen 3.000 und 3.500 Euro bewegen. Mithin liegt der Gedanke nahe, die schon jetzt existierende Deckungslücke ganz oder teilweise durch eine private, kapitalgedeckte Pflegetagegeldversicherung zu schließen, wie sie viele Versicherungsunternehmen anbieten. Die politische Entscheidungsfrage lautet dabei, ob der Staat eine solche Zusatzversicherung subventionieren oder sogar obligatorisch machen soll und in welchem Verhältnis die umlagefinanzierte und die kapitalgedeckte Absicherung des Pflegerisikos in der Zukunft stehen sollen, um mehr Nachhaltigkeit zu erreichen. Einen Überblick über verschiedene Modelle geben Arentz et al. (2011).

Die schwarz-gelbe Bundesregierung hatte sich im Koalitionsvertrag von 2009 darauf festgelegt, die Pflegeversicherung um einen kapitalgedeckten Teil zu ergänzen, der „verpflichtend, individualisiert und generationengerecht“ sein sollte (Koalitionsvertrag 2009, S. 93). Die 2012 im Bundestag verabschiedete Pflegereform bedeutete jedoch den Abschied von der Idee eines Obligatoriums. Stattdessen werden seit dem 1. Januar 2013 private Pflegetagegeldversicherungen mit bis zu 60 Euro im Jahr vom Staat bezuschusst („Pflege-Bahr“). Eine solche Lösung ist wegen der Mitnahme-Effekte problematisch. Einen Vertrag schließen vor allem Besserverdienende freiwillig, bei denen die Wahrscheinlichkeit der späteren Inanspruchnahme von Sozialhilfe ohnehin gering ist. Bei diesen spielt auch der staatliche Zuschuss keine entscheidende Rolle.

Die ersten Erfahrungen mit dem Programm scheinen diese Skepsis zu bestätigen: Bis zum Jahresende 2014 waren lediglich etwa 550.000 Verträge abgeschlossen worden'. Das sind kaum mehr als 1,5 Prozent der Wohnbevölkerung in der Altersgruppe zwischen 30 und 60 Jahren

9 Schriftliche Auskunft des Bundesgesundheitsministeriums vom 9. Februar 2015. (etwa 35 Millionen), für die eine private Zusatzvorsorge besonders sinnvoll erscheint. Entsprechend gering ist der Beitrag dieser Maßnahme zur Steigerung der Nachhaltigkeit der Finanzierung.

In einem weiteren Reformschritt sollte der Gesetzgeber daher die Zusatzversicherung für obligatorisch erklären und seine Zuschüsse auf den Kreis jener Personen beschränken, welche die entsprechenden Beiträge aus dem eigenen Einkommen nicht aufbringen können. Wenn man beispielsweise für die heute mittlere Generation (zwischen 30 und 60 Jahren) die Hälfte der derzeitigen Deckungslücke von 1.800 Euro im Monat in der Pflegestufe 3 schließen wollte, müssten Angehörige der genannten Altersgruppen verpflichtet werden, eine Pflegetagegeldversicherung über 900 Euro im Monat in Pflegestufe 3 (und gestaffelte Leistungen in den unteren Stufen) abzuschließen. Solche Verträge werden derzeit je nach Eintrittsalter für Monatsbeiträge von etwa 15 Euro für 30-jährige, etwa 30 Euro für 45jährige und etwa 60 Euro für 60-jährige Personen angeboten. ${ }^{10}$ Wenn alle 35 Millionen Personen der genannten Altersgruppe einen entsprechenden Vertrag abschlössen, ergäbe das eine Kapitalbildung von etwa 12 Milliarden Euro im Jahr. Diese Kapitalbildung erfolgte allerdings nicht notwendigerweise zusätzlich, sondern würde teilweise andere Sparformen verdrängen. Im Unterschied zu den RiesterVerträgen würden jedoch die Leistungen ausschließlich bei den Versicherten anfallen, die in Folge von Pflegebedürftigkeit einen besonderen Finanzbedarf haben. Sie wäre darauf ausgerichtet, künftige Ansprüche auf Sozialhilfe zu ersetzen und damit die nachfolgenden Generationen an Steuerzahlern zu entlasten.

\subsection{Zur Durchsetzbarkeit grundlegender Reformen in alternden Gesellschaften}

Wem nützt Nachhaltigkeit der Finanzierung eigentlich? Sind es die nachfolgenden Generationen von Beitrags- und Steuerzahlern, die vor einer Überforderung geschützt werden sollen - worauf der Begriff der „Generationengerechtigkeit“ hindeutet? Oder sind es eher die „Alten“, deren gesetzliche Ansprüche auf eine auskömmliche Rente, auf medizinische Behandlung nach dem Stand des Wissens und auf notwendige Pflegeleistungen nicht oder nur partiell erfüllt werden? Die Antwort hängt davon ab, ob der angesprochene Anstieg der Beitragssätze sowie die ent-

10 Tarife online unter www.pflegetagegeldversicherung.com, abgerufen am 21.1.2015. Es handelt sich bei den genannten Werten um den Tarif des Testsiegers DFV mit 600 Euro Demenzgeld, Dynamik ohne Gesundheitsprüfung und Beitragsfreiheit bei Pflegebedürftigkeit. 
sprechenden steuerfinanzierten Sozialleistungen das Parlament passieren oder ob dies politisch verhindert wird mit der Folge, dass Leistungsversprechen zurückgenommen werden müssen.

Sinn und Übelmesser (2002) warnen, dass Reformen zu Lasten der Rentnergeneration in einer Mehrheits-Demokratie nicht mehr möglich seien, sobald diese die Mehrheit der wahlberechtigten Bürger stelle. Die Kosten einer nichtnachhaltigen Finanzierung müssten demnach die späteren Generationen tragen. Dieser Einschätzung widersprechen Breyer und Stolte (2001) mit dem Argument, dass die mittlere Generation, die das Sozialprodukt erwirtschaftet, andere Mittel als das Stimmrecht an der Wahlurne besitzt, um ihre Interessen durchzusetzen. Sie reichen vom Rückzug in die Selbstständigkeit (bei lohnbezogener Erhebung von Sozialabgaben) über politisch motivierte Streiks bis hin zur Auswanderung. Und wenn eine Rentnergeneration, die mit der gesamten politischen Macht ausgestattet ist, diese tatsächlich so einsetzen sollte, dass sie die maximalen Sozialleistungen für sich erzielt, dann bedeutet dies nicht, dass es zu einem maximalen Beitragssatz kommt. Vielmehr dürfte jener Satz gewählt werden, der das Beitragsaufkommen maximiert, und zwar unter Beachtung von Abgabenwiderständen.

Aus dieser Überlegung folgt, dass nicht die jungen, sondern die alten Generationen den Schaden haben, wenn die Finanzierung des Sozialsystems nicht nachhaltig ist. Deren gesetzlich verankerte Leistungsansprüche müssten dann wegen mangelnder Finanzierbarkeit zurückgeschraubt werden, so wie bereits in den Rentenreformen zwischen 2001 und 2007 zuvor bestehende Leistungsversprechen zurückgenommen wurden. Folglich sind es gerade die heutigen Beitragszahler und morgigen Rentner, die am meisten von Reformen zur Stärkung der Nachhaltigkeit der Finanzierung profitieren.

\section{Fazit: Beschleunigtes Ausgabenwachstum}

Nach einer langen und kontroversen Diskussion in der Literatur darf man davon ausgehen, dass die demographische Alterung in Deutschland das Wachstum der Pro-KopfAusgaben in der Gesetzlichen Krankenversicherung weiter beschleunigen wird. In die gleiche Richtung - und gegebenenfalls noch stärker - wird sich der medizinische Fortschritt auswirken. Noch gravierender als in der Krankenversicherung wird der Anstieg der Ausgaben in der sozialen Pflegeversicherung sein, vor allem dann, wenn das gegenwärtig unzureichende Leistungsniveau in Zukunft angeho- ben werden soll. Eine Umschichtung in der Struktur des Bruttoinlandsprodukts zugunsten von Gesundheits- und Pflegeausgaben muss nicht per se schlecht sein, wenn den höheren Ausgaben eine höhere Produktqualität gegenübersteht. Problematisch ist jedoch, dass die Ausgaben zum weit überwiegenden Teil durch Zwangsabgaben finanziert werden, die obendrein eine starke intergenerative Transferkomponente beinhalten.

Danksagung: Für wertvolle Hinweise danke ich Stefan Felder (Universität Basel) und Karl-Heinz Paqué sowie für eine signifikante stilistische Verbesserung Karen Horn.

\section{Literatur}

Arentz, C., Läufer, I. und Roth, S.J. (2011), Reform der gesetzlichen Pflegeversicherung: Teilkapitaldeckungsmodelle im Vergleich, Otto-Wolff-Institut Discussion Paper 04.

Atella, V. und V. Conti (2013), The effect of age and time to death on health care expenditures: The Italian experience, CEIS Tor Vergata Research Paper Series 11(4), Nr. 267, März 2013.

Barros, P.P. (1998), The black box of health care expenditure growth determinants, Health Economics 7, S. 533-44.

Bjørner, T.B. und S. Arnberg (2012), Terminal costs, improved life expectancy and future public health expenditure, International Journal of Health Care Finance and Economics 12, S. 129-43.

Breyer, F. (2004), Pflege und Gesundheit, in: P. Masuch et al. (Hrsg.), Grundlagen und Herausforderungen des Sozialstaats. Denkschrift 60 Jahre Bundessozialgericht, Erich Schmidt Verlag, Berlin, S. 729-49.

Breyer, F. (2012), Legale und illegale Wege zu einer Bürgerversicherung, Wirtschaftsdienst 92, Heft 10, S. 655-58.

Breyer, F. (2013), Wettbewerb als Steuerungsform im Gesundheitswesen, Public Health Forum 21, S. 3-5.

Breyer, F. und S. Felder (2006), Life expectancy and health care expenditures: A new calculation for Germany using the costs of dying, Health Policy 75, S. 178-86.

Breyer, F., N. Lorenz und T. Niebel (2015), Population ageing and health care expenditures: Is there a Eubie Blake effect?, European Journal of Health Economics 16, S. 95-112.

Breyer, F. und K. Stolte (2001), Demographic change, endogenous labor supply, and the feasibility of pension reform, Journal of Population Economics 14, S. 409-24.

Breyer, F. und V. Ulrich (2000), Gesundheitsausgaben, Alter und medizinischer Fortschritt: eine Regressionsanalyse, Jahrbücher für Nationalökonomie und Statistik 220, S. 1-17.

Bundesministerium für Gesundheit und Soziale Sicherheit (Hrsg.) (2003), Nachhaltigkeit in der Finanzierung der Sozialen Sicherungssysteme, Bericht der Kommission, Berlin.

Callahan, D. (1987), Setting Limits. Medical Goals in an Aging Society, Simon \& Schuster, New York.

Cassel, D. und V. Oberdieck (2002), Kapitaldeckung in der Gesetzlichen Krankenversicherung, Wirtschaftsdienst 82, S. $15-22$.

Colombier, C. und W. Weber (2011), Projecting health-care expenditure for Switzerland: further evidence against the "red herring" 
hypothesis, International Journal of Health Planning and Management 26, S. 246-63.

Comas-Herrera, A., R. Wittenberg, J. Costa-Font, C. Gori, A. di Maio, C. Patxot, L. Pickard, A. Pozzi und H. Rothgang (2006), Future long-term care expenditure in Germany, Spain, Italy and the United Kingdom, Ageing and Society 26, S. 285-302.

Daniels, N. (1985), Just Health Care, Cambridge University Press, Cambridge.

De Meijer, C., M. Koopmanschap, T. Bago d'Uva und E. van Doorslaer (2009), Time to drop time-to-death? Unraveling the determinants of LTC spending in the Netherlands, Netspar Discussion Paper 11/2009-045.

Dormont, B., M. Grignon und H. Huber (2006), Health expenditure growth: reassessing the threat of ageing, Health Economics 15, S. 947-63.

Felder, S., M. Meier und H. Schmitt (2000), Health care expenditure in the last months of life, Journal of Health Economics 19, S. 67995.

Felder, S., A. Werblow und P. Zweifel (2010), Do red herrings swim in circles? Controlling for the endogeneity of time to death, Journal of Health Economics 29, S. 205-12.

Fries, J.F. (1980), Ageing, natural death, and the compression of morbidity, New England Journal of Medicine 303, S. 130-6.

Fuchs, V.R. (1984), Though much is taken: Reflections on aging, health and medical care, Milbank Memorial Fund Quarterly/ Health and Society 62, S. 143-66.

Geue, C., A. Briggs, J. Lewsey und P. Lorgelly (2014), Population ageing and healthcare expenditure projections: New evidence from a time to death approach, European Journal of Health Economics 15: 885-96.

Gregersen, F.A. (2014), The impact of ageing on health care expenditures: A study of steepening, European Journal of Health Economics 15, S. 979-89.

Gregersen, F.A. und G. Godager (2013), Hospital expenditures and the red herring hypothesis: Evidence from a complete national registry, University of Oslo HERO Working Paper 3.

Greß, S. und K.-J. Bieback (2013), Zur Umsetzbarkeit einer Bürgerversicherung bei Krankheit und Pflegebedürftigkeit, Gutachten für den Arbeiterwohlfahrt Bundesverband, Mai.

Häcker, J., T. Hackmann und B. Raffelhüschen (2011), Soziale Pflegeversicherung heute und morgen, Deutsches Institut für Altersvorsorge, Köln.

Hall, R.E. und C.I. Jones (2007), The value of life and the rise in health spending, Quarterly Journal of Economics 122, S. 39-72.

Karlsson, M. und F. Klohn (2014), Testing the red herring hypothesis on an aggregated level: Ageing, time-to-death and care costs for older people in Sweden, European Journal of Health Economics 15 , S. 533-51.

Koalitionsvertrag zwischen CDU, CSU und FDP, 17. Legislaturperiode (2009), Bildung. Wachstum. Zusammenhalt, Berlin. Online verfügbar unter: http://www.fdp.de/files/565/091024-koalitions vertrag.pdf

Krämer, W. (1993), Wir kurieren uns zu Tode. Die Zukunft der modernen Medizin, Campus, Frankfurt/New York.

Lubitz, J.D., J. Beebe und C. Baker (1995), Longevity and medicare expenditure, New England Journal of Medicine 332, S. 9991003.

Lubitz, J.D. und G.F. Riley (1993), Trends in medicare payments in the last year of life, New England Journal of Medicine 328, S. 1092-96.
Miller, T. (2001), Increasing longevity and medicare expenditures, Demography 38, S. 215-26.

OECD (2013), Health at a Glance 2013: OECD Indicators, OECD Publishing, Paris.

Olshansky, S.J., M.A. Rudberg, B.A. Carnes, C.K. Cassel und J.A. Brody (1991), Trading off longer life for worsening health: The expansion of morbidity hypothesis, Journal of Aging and Health 3 , S. $194-216$.

Polder, J.J., J.J. Barendregt und H. van Oers (2006), Health care costs in the last year of life - the Dutch experience, Social Science and Medicine 63, S. 1720-31.

Rothgang, H. (2011), Reformoptionen und Reformsackgassen, Wirtschaftsdienst 91, S. 659-63.

Salas, C. und J.P. Raftery (2001), Econometric issues in testing the age neutrality of health care expenditure, Health Economics 10, S. 669-71.

Schmitz, H. und N.R. Ziebarth (2011), In absolute or relative terms? How framing prices affects the consumer price sensitivity of health plan choice, IZA Discussion Paper Nr. 6241, Dezember.

Seshamani, M. und A. Gray (2004a), Ageing and health care expenditure: The red herring argument revisited, Health Economics 13, S. 303-14.

Seshamani, M. und A. Gray (2004b), A longitudinal study of the effects of age and time to death on hospital costs, Journal of Health Economics 23, S. 217-35.

Shang, B. und D. Goldman (2008), Does age or life expectancy better predict health care expenditures?, Health Economics 17, S. 487-501.

Sinn, H.-W. und S. Übelmesser (2002), Pensions and the path to gerontocracy in Germany, European Journal of Political Economy 19, S. 153-58.

Statistisches Bundesamt (2015a), Bevölkerung Deutschlands bis 2060, 13. koordinierte Bevölkerungsvorausberechnung, Wiesbaden.

Statistisches Bundesamt (2015b), Ausgaben der Sozialhilfe - Hilfe zur Pflege. Online verfügbar unter:

Statistisches Bundesamt, div. Jahrgänge, Pflegestatistik 20xx, Wiesbaden.

Stearns, S.C. und E.C. Norton (2004), Time to include time to death? The future of health care expenditure predictions, Health Economics 13, S. 315-27.

Steinmann, L., H. Telser und P. Zweifel (2007), Aging and future healthcare expenditure: A consistent approach, Forum for Health Economics and Policy 10/2, Artikel 1.

Strech, D., M. Synofzik und G. Marckmann (2008), How physicians allocate scarce resources at the bedside: A systematic review of qualitative studies, Journal of Medicine and Philosophy 33, S. 80-99.

Van Baal, P.H. und A. Wong (2012), Time to death and the forecasting of macro-level health care expenditures: Some further considerations, Journal of Health Economics 31, S. 876-87.

Werblow, A., S. Felder und P. Zweifel (2007), Population ageing and health care expenditure: A school of "red herrings"?, Health Economics 16, S. 1109-26.

Werding, M. (2013): Alterssicherung, Arbeitsmarktdynamik, und neue Reformen: Wie das Rentensystem stabilisiert werden kann, Studie im Auftrag der Bertelsmann-Stiftung, Gütersloh. Online verfügbar unter: http://www.bertelsmann-stiftung.de/cps/rde/ xbcr/SID-AF257E48-76C67700/bst/xcms_bst_dms_37459_ 37732_2.pdf. 
Wong, A., P.H.M. van Baal, H.C. Boshuizen und J.J. Polder (2011), Exploring the influence of proximity to death on disease-specific hospital expenditures: A carpaccio of red herrings, Health Economics 20, S. 379-400.

Zweifel, P., S. Felder und M. Meier (1999), Ageing of population and health care expenditure: A red herring?, Health Economics 8, S. 485-96.

Zweifel, P., S. Felder und A. Werblow (2004), Population ageing and health care expenditure: New evidence on the "red herrings", The Geneva Papers on Risk and Insurance 29, S. 652-66.

Zweifel, P. und M. Ferrari (1992), Is there a Sisyphus syndrome in health care? In: P. Zweifel und H.E. Frech (Hrsg.), Health Economics Worldwide, Kluwer, Boston/Dordrecht, S. 311-30.

Zweifel, P., L. Steinmann, und P. Eugster (2005), The Sisyphus syndrome in health revisited, International Journal of Health Care Economics and Financing 5, S. 127-45.

\section{Autoreninformation}

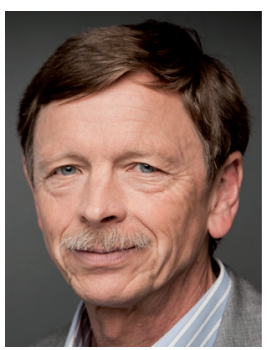

Prof. Dr. Friedrich Breyer

Lehrstuhl für Wirtschafts- und

Sozialpolitik, Universität Konstanz,

D-78457 Konstanz

friedrich.breyer@uni-konstanz.de

Friedrich Breyer (geb. 1950) ist seit 1992 Ordinarius für Volkswirtschaftslehre an der Universität Konstanz und seit 2000 zudem Forschungsprofessor am Deutschen Institut für Wirtschaftsforschung, Berlin. Er ist Mitglied des Wissenschaftlichen Beirats beim Bundesministerium für Wirtschaft und Technologie, war 2008 Mitbegründer der Deutschen Gesellschaft für Gesundheitsökonomie (DGGÖ) und diente ihr 2012/13 als Vorsitzender. Seine Hauptarbeitsgebiete sind die Ökonomie der Sozialen Sicherung und die Gesundheitsökonomie. Er ist Autor von mehr als 60 Aufsätzen in referierten Fachzeitschriften und zahlreichen Lehrbüchern, darunter dem Standardwerk Gesundheitsökonomik, 6. Auflage 2012 (mit Peter Zweifel und Mathias Kifmann). 